\title{
Functional mitral regurgitation causing severe dysphagia resolved with isolated aortic valve replacement: an unusual case with few learning points
}

\author{
Kumar Rajanikant ${ }^{1}$, Ajay Kumar ${ }^{2 *}$, Rinkesh Bansal ${ }^{3}$, Rajiv Juneja ${ }^{4}$
}

\begin{abstract}
${ }^{1}$ Senior Resident, ${ }^{\mathbf{2}}$ Associate Professor, ${ }^{\mathbf{3}, 4}$ Senior Consultant, ${ }^{\mathbf{1}, 2,4}$ Dept. of Anaesthesia, ${ }^{3}$ Dept. of Gastroenterology, ${ }^{1}$ Post Graduate Institute of Medical Education \& Research, Chandigarh, ${ }^{\mathbf{2}}$ All India Institute of Medical Sciences, Rishikesh, Uttarakhand, ${ }^{\mathbf{3}, 4}$ Medanta, The Medicity, Gurugram, Haryana, India
\end{abstract}

*Corresponding Author: Ajay Kumar

Email: ajaymishra0701@gmail.com

Received: $21^{\text {st }}$ January, 2019

Accepted: $20^{\text {th }}$ March, 2019

\begin{abstract}
Dysphagia due to compression of esophagus by enlarged left atrium (LA) due to mitral valve pathology is known. However, a functional mitral regurgitation (MR) as a consequence of aortic stenosis (AS) causing LA enlargement and dysphagia is rare and probably not reported. We present a case in which dysphagia was caused by functional MR secondary to AS. Dysphagia resolved significantly after isolated aortic valve replacement within postoperative hospitalization period and completely with improvement in diastolic function as evidenced by follow up echocardiographic examinations. Intraoperative transesophageal echocardiography was also used without any complication despite the patient having dysphagia after conducting preliminary endoscopic examination.
\end{abstract}

Keywords: Dysphagia, Left atrial enlargement, Mitral regurgitation, Valve replacement.

\section{Introduction}

Left atrium of the heart lies in the midline and enlargement occurs in the direction of least resistance i.e. posteriorly and pushes the esophagus against vertebrae. This can cause dysphagia. However, cardiovascular disease is one of the least common cause of dysphagia. ${ }^{1}$ Left atrial enlargement (LAE) is caused by rheumatic mitral stenosis, mitral regurgitation (MR) and paravalvular leak in prosthetic mitral valve. ${ }^{2}$ Functional MR causing dysphagia is far more uncommon and probably not reported in literature. We describe a case, in which LAE due to functional MR secondary to aortic stenosis (AS) was the cause of dysphagia. Isolated aortic valve replacement (AVR) led to resolution of dysphagia, decrease in severity of MR and LA chamber size during a six month follow up. A written and informed consent has been obtained from the patient to publish details of his treatment.

\section{Case}

A 55 year old male weighing $55 \mathrm{Kg}$ and height of 1.6 $\mathrm{m}$ presented with progressively increasing dyspnea on exertion (New York Heart Association class III) and pitting edema for the last one year. On further workup he was diagnosed to have severe aortic stenosis with left ventricular dysfunction. He was advised to undergo aortic valve replacement. He was administered torsemide $10 \mathrm{mg}$ OD, Carvedilol $3.125 \mathrm{mg}$ OD to alleviate his congestive signs and symptoms. During admission to the hospital for valve replacement surgery, he skipped his torsemide tablets and developed dysphagia to solids and cough on swallowing liquids.

On examination, he had regular but feeble pulse of $74 / \mathrm{min}$, blood pressure of $87 / 74 \mathrm{mmHg}$. Due to his poor hemodynamic condition, sudden onset of dysphagia, he was shifted to intensive care. There was normal vesicular breath sounds on auscultation. Transthoracic echocardiography (TTE) re-established previous diagnosis of calcific aortic valve with severe AS (valve area $0.58 \mathrm{~cm}^{2}$ ), high normal left ventricular size (diastolic: systolic diameter: 52/48 mm), mild aortic regurgitation (AR), moderate to severe mitral regurgitation (MR), moderate tricuspid regurgitation (TR). It also revealed diastolic dysfunction, raised left ventricular end diastolic pressure (LVEDP) (Table 1), bi-atrial enlargement, severe right ventricular (RV) systolic dysfunction, pulmonary artery hypertension and a left ventricular ejection fraction $(\mathrm{EF})$ of $20 \%$.

Contrast computed tomography (CT) of the thorax showed calcified aortic valve and LA abutting the oesophagus (Fig. 1a). No abnormal growth was seen in or around the esophagus. On barium swallow, smooth extrinsic indentation was noted on the lower anterior esophageal wall, that lead to a thin rat tail flow appearance of barium through the rest of the oesophagus to the stomach (Fig. 1b). Upper gastrointestinal (GI) endoscopy revealed a bulge in the lumen of the anterior wall of the lower esophagus with smooth mucosa with no growth or stricture. (Supposedly due to LA lying anteriorly (Fig. 1c). Since he was hemodynamically unstable, esophageal manometry was postponed. Normal endoscopic contour of esophagus paved the way for intraoperative use of TEE. Intraoperative TEE was necessary to decide whether to intervene mitral valve or not. Intraoperative TEE revealed moderate MR with large LA volume (104 ml) (Fig. 1d) normal MV cusps and anatomy, vena contracta of $0.36 \mathrm{~cm}$, mitral regurgitant area to be $25 \%$ of LA area and preserved left ventricular contractility $(\mathrm{dP} / \mathrm{dT}=1136 \mathrm{mmHg} / \mathrm{sec})($ Video 1$)$. It was decided to proceed with AVR only to cut short the ischemia time and time spent on cardiopulmonary bypass. A St. Jude size $23 \mathrm{~mm}$ mechanical valve prosthesis was secured at the aortic valve position. 
In the OR, Patient was induced with etomidate 0.3 $\mathrm{mg} / \mathrm{kg}$. His pulmonary artery pressure at the time of induction was 50/24(34) $\mathrm{mmHg}$, PAWP of $22 \mathrm{mmHg}$. Prior endoscopy had paved the way for safe insertion of TEE probe to assess whether mitral and tricuspid valves need to be operated. Intraoperative TEE endorsed the transthoracic echocardiography findings but mitral and tricuspid regurgitation (PASP of $28 \mathrm{mmHg}$ ) was found to be mild with normal coaptation of mitral leaflet. The patient was hemodynamically unstable in the immediate postoperative period due to poor myocardial reserve and was shifted to the intensive care unit on three Inotropes IV Milrinone 0.5 $\mu \mathrm{gm} / \mathrm{kg} / \mathrm{hr}$, IV Adrenaline $0.1 \mu \mathrm{gm} / \mathrm{kg} / \mathrm{hr}$, IV Noradrenaline $0.1 \mu \mathrm{gm} / \mathrm{kg} /$ and an intraaortic balloon pump (IABP). Postoperatively his mean pulmonary artery pressure (mPAP) decreased from $40 \mathrm{mmHg}$ to $23 \mathrm{mmHg}$, cardiac index increased from $0.8 \mathrm{~L} / \mathrm{m}^{2}$ to $1.5 \mathrm{~L} / \mathrm{m}^{2}$ by day 2 . The patient was extubated on post-operative day 2 ; IABP was removed on day 3. Surprisingly, he had no complaints of dysphagia to solids or liquids after extubation.

At 3 month follow up, he acknowledged improvement in functional state to NYHA I. TTE affirmed improved LVEF to $40 \%$ and improvement of diastolic function from restrictive pattern to pseudonormal filling [Table 1]. There was a mild MR, normal RV function (Tricuspid Annular Systolic Velocity- $10 \mathrm{~cm} / \mathrm{sec}$ ), mild TR. Though myocardial function had improved, LVEDP remained on moderately higher side, as shown by high E/e' ratio (Table 1). The amelioration in mitral regurgitation from moderate to mild, improved diastolic function, decrease in left ventricular end diastolic diameter from 52 to $42 \mathrm{~mm}$ was noted at six months follow-up [Table 1].
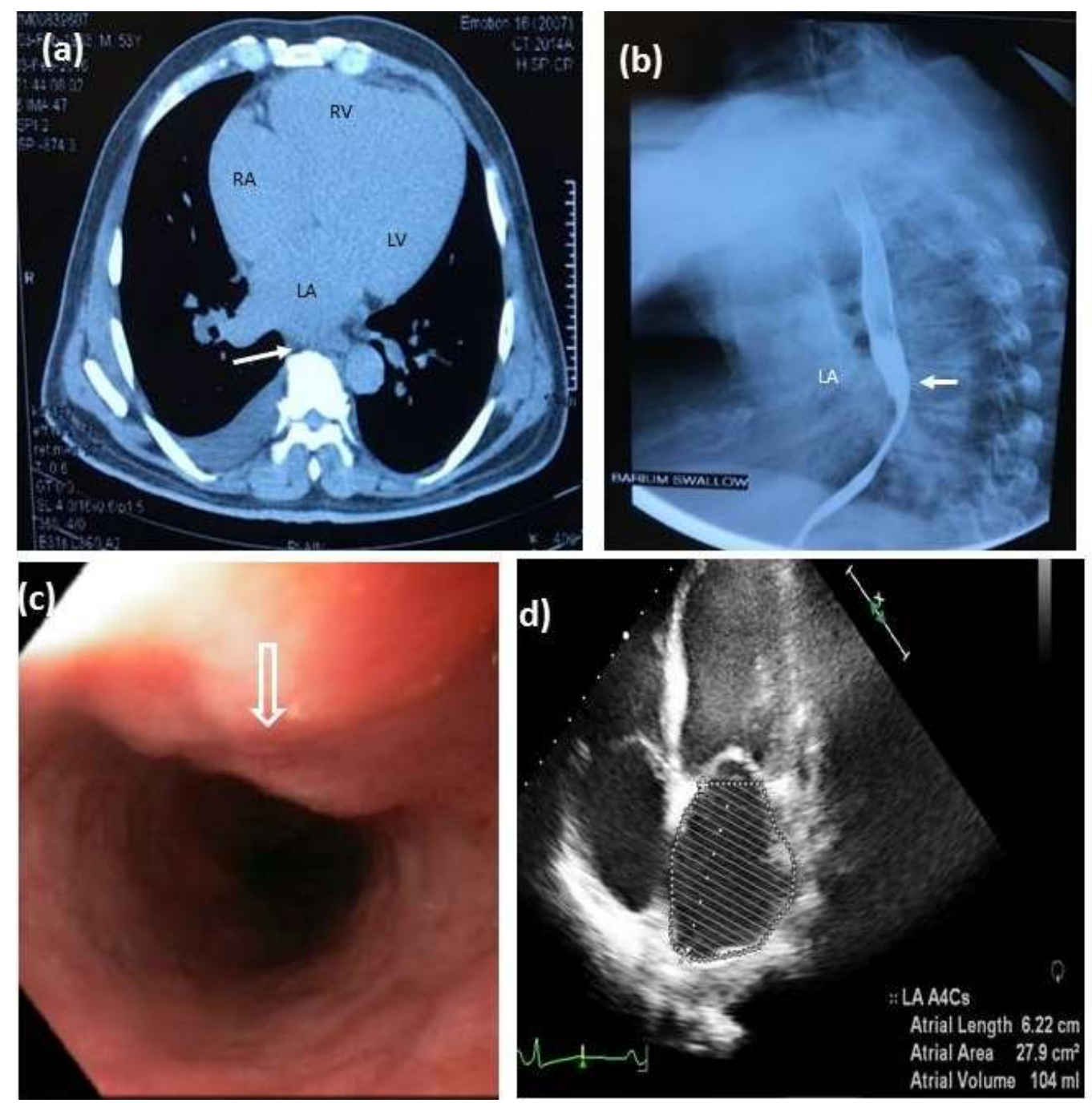

Fig. 1 (a): Contrast CT showing compression of oesophagus against body of spine (White arrow); (b): Lateral radiograph depicting rat tail appearance of barium meal swallow (Solid arrow); (c): Oesophagoscopy shows bulging of left atrial wall into the oesophagus (Hollow arrow); (d): Transthoracic echocardiographic apical four chamber view showing enlarged left atrium in preoperative evaluation 
Table 1: Echocardiographic parameters in perioperative and follow-up period

\begin{tabular}{|l|c|c|c|c|}
\hline \multicolumn{1}{|c|}{ Parameters } & Preoperative & Postoperative & At 3 months & At 6 months \\
\hline LVEF $(\%)$ & 20 & 28 & 42 & 47 \\
\hline AV gradient $(\mathrm{mmHg})$ & & & & \\
Peak & 56 & 13 & 13 & 13 \\
Mean & 41 & 7 & 7 & 7 \\
\hline LA $(\mathrm{ml})$ & 104 & 104 & 66 & 57 \\
\hline LVEDD $(\mathrm{mm})$ & 52 & 52 & 48 & 42 \\
\hline E $(\mathrm{cm} / \mathrm{sec})$ & 122 & 122 & 63 & 55 \\
\hline A $(\mathrm{cm} / \mathrm{sec})$ & 36 & 51 & 75 & 100 \\
\hline E/A ratio & 3.4 & 3.0 & 0.85 & 0.55 \\
\hline E/e' ratio & 40 & 40 & 21 & 11 \\
\hline PASP $(\mathrm{mmHg})$ & 50 & 32 & 29 & 18 \\
\hline
\end{tabular}

(LVEF- left ventricular ejection fraction; AV- aortic valve; LA- left atrium; ERO- effective regurgitant orifice area; E- early diastolic flow across mitral valve; A- late diastolic flow across mitral valve; E/A ratio; E/e' ratio- ratio of mitral peak velocity of early filling to early diastolic mitral annular velocity, PASP- pulmonary artery systolic pressure, LVEDD---Left Ventricular End Diastolic Diameter, LVEF Left Ventricular Ejection Fraction)

\section{Discussion}

In this case LAE was due to moderate to severe functional secondary MR. Severe AS led to ventricular dilatation and systolic dysfunction. Obstruction of aortic outflow augmented the volume of MR across the dilated MV annulus, the MR itself caused decreased preload, which is vital for left ventricular stroke volume in patients with aortic stenosis. ${ }^{3}$ This led to decreased cardiac output, LAE and increased pulmonary venous hypertension. As evident in this case which also had a high normal left ventricle end diastolic diameter $(52 \mathrm{~mm})$, high pulmonary artery pressure and low cardiac index $\left(0.8 \mathrm{~L} / \mathrm{m}^{2}\right)$. The regurgitation in functional MR results from failure of coaptation of the mitral valve leaflets unlike the primary MR where coexisting structural changes of the valve leads to regurgitation. ${ }^{4}$

Dysphagia due to LAE was critically analyzed for the first time by Tinney and Schmidt in $1943 .{ }^{1}$ LAE leads to localized high pressure in the mid oesophagus and pulsations correlate with QRS complexes of the electrocardiogram. ${ }^{2}$ Indirect evidence of LAE causing dysphagia includes chest $\mathrm{x}$-ray demonstrating a double atrial shadow, barium meal showing transient hold up, CT angiography showing compression of esophagus against the body of spine, and endoscopy showing bulge in the anterior wall at mid esophagus level. ${ }^{5}$ These were all present in this case. Manometric evidence of increased oesophageal pressure has no clear relationship with oesophagographic findings of dysphagia. However marked increase in oesophageal pressure $>11 \mathrm{mmHg}$ and absence of relaxation in response to swallowing indicates evidence of cardiovascular dysphagia. ${ }^{6}$ We could not measure oesophageal pressure as patient was unable to tolerate further procedures. Since there was no other cause for dysphagia and it improved after AVR it could be assumed that increased left atrial pressure due to functional MR caused dysphagia in this patient. The mPAP pressure decreased from 40 to 23 from preoperative to postoperative state, which is also a surrogate of decreased LAP in type 2 pulmonary hypertension after therapeutic intervention. ${ }^{7}$ To the best of our knowledge this is the first report of LAE causing dysphagia due to secondary MR.

Concomitant MV repair or replacement with AVR is recommended in severe secondary MR and also moderate MR in patients undergoing other cardiac surgery (class of recommendation II b, level of evidence C).${ }^{8,9}$ However the guidelines also state the lack of strong evidence that MV replacement or repair improves prognosis in secondary MR despite the apparent prognostic significance of lower effective regurgitant orifice area and right ventricular volumes. ${ }^{10}$

Study suggest improvement in severe and moderate functional MR in $82 \%$ of the patients undergoing AVR without correction of functional MR. ${ }^{11}$ Even in cases of thoracic endovascular AVR, most of the patients with high grade MR demonstrated substantial improvement in MR grade (47\% in severe MR and $35 \%$ in moderate MR) at 1 year without correction. ${ }^{12}$ Proponents of isolated AVR have justified the conservative approach towards mitral valve citing onset of reverse remodeling after surgery which reduces functional MR. ${ }^{11}$ Replacement of both aortic and mitral valves significantly increases aortic cross clamp and bypass time, surgical morbidity and mortality. Considering the above facts and since intraoperative TEE demonstrated normal cusps without structural disease of MV, moderate to severe MR, and poor myocardial function, mitral valve intervention was not done in an effort to minimize morbidity.

The mechanism in improvement of MR following AVR and hence dysphagia was multifactorial. Firstly improved aortic valve performance with a decrease in afterload reduces pathological retrograde blood flow into LA. Secondly, the neurohormonal response caused by heart failure state is reduced. Thirdly, restoration of proper geometry of left ventricle contributes to improved function of the mitral valve apparatus. Reverse remodeling sets in early and is responsible for decrease in left ventricular end diastolic volume, mitral annular diameter and mitral 
regurgitation, ${ }^{11,12}$ as was observed by a significant change in diastolic function at six month follow-up.

Dysphagia is a relative contraindication to TEE. ${ }^{13}$ However, intraoperative TEE was important and played a significant role in this case for decision-making. An upper GI endoscopy (which is recommended in dysphagia), ${ }^{14}$ confirmed a smooth esophageal mucosa without evidence of internal growth, obstruction or stricture which enabled an uneventful TEE.

This case demonstrates few facts for us. Although rare, severe dysphagia may be present in AS patient caused by LAE due to functional MR. It reiterates a conservative approach towards moderate to severe functional mitral regurgitation in case of aortic valve disease with structurally normal $\mathrm{MV}$, and the value of pre-procedure upper GI endoscopy in cases of relative contraindication to TEE.

\section{Conflict of Interest: None.}

\section{References}

1. Behl PR, Holden MP. Mitral valve disease and dysphagia. Eur Heart J 1984;5:919-23.

2. Cappell MS. Endoscopic, radiographic, and manometric findings associated with cardiovascular dysphagia. Dig Dis Sci 1995;40:166-76.

3. Bonow RO, DL Mann, DP Zipes, P Libby. Valvular heart disease. In: Bonow RO, Mann DL, Zipes DP, Libby P (editors). Braunwald's Heart Diesease. $9^{\text {th }}$ ed. Philadelphia: Saunders Elsevier, Inc; 2011.pp.1468-1530.

4. Schmitto JD, Lee LS, Mokashi SA, Bolman RM, Cohn LH, Chen FY. Functional mitral regurgitation. Cardiol Rev 2010;18:285-91.

5. Krishan RK, Tandon R. Rheumatic fever and rheumatic heart disease: last 50 years. Indian J Med Res 2013;137:643-58.

6. Stagias JG, Ciarolla D, Campo S, Burrell MI, Traube M. Vascular compression of the esophagus: a manometric and radiologic study. Dig Dis Sci 1994;39:782-6.

7. Galiè N, Humbert M, Vachiery JL, Gibbs S, Lang I, Torbicki A et al.. 2015 ESC/ERS Guidelines for the diagnosis and treatment of pulmonary hypertension: The Joint Task Force for the Diagnosis and Treatment of Pulmonary Hypertension of the European Society of Cardiology (ESC) and the European Respiratory Society (ERS). Eur Heart J 2016 ;37:67-119.

8. Barreiro CJ, Patel ND, Fitton TP. Aortic valve replacement and concomitant mitral valve regurgitation in the elderly: impact on survival and functional outcome. Circulation 2005;112(9 Suppl):I443-7.

9. Nishimura RA, Otto CM, Bonow RO. 2014 AHA/ACC guideline for the management of patients with valvular heart disease: a report of the American College of

Cardiology/American Heart Association Task Force on Practice Guidelines. J Thorac Cardiovasc Surg 2014;148(1):e1-e132.

10. Nishimura RA, Otto CM, Bonow RO, Carabello BA, Erwin JP 3rd, Fleisher LA, et al. 2017 AHA/ACC Focused Update of the 2014 AHA/ACC Guideline for the Management of Patients With Valvular Heart Disease: A Report of the American College of Cardiology/American Heart Association Task Force on Clinical Practice Guidelines. Circ 2017;135(25):e1159e1195.

11. Unger P, Plein D, Van Camp G, Cosyns B, Pasquet A, Henrard $\mathrm{V}$, et al. Effects of valve replacement for aortic stenosis on mitral regurgitation. Am J Cardiol 2008;102:1378-82.

12. Bedogni F, Latib A, De Marco F, Agnifili M, Oreglia J, Pizzocri S, et al. Interplay between Mitral Regurgitation and Transcatheter Aortic Valve Replacement With the Core Valve Revalving System. Circ 2013;128:2145-53.

13. Hilberath JN, Oakes DA, Shernan SK, Bulwer BE, D'Ambra MN, Eltzschig HK. Safety of Transesophageal Echocardiography. J Am Soc Echocardiogr 2010;23:1115-27.

14. Garcia-Tsao G, Sanyal AJ, Grace ND, Carey WD. Prevention and management of gastroesophageal varices and variceal hemorrhage in cirrhosis. Am J Gastroenterol 2007;102:2086102.

How to cite this article: Rajanikant $\mathrm{K}$, Kumar A, Bansal R, Juneja R. Functional mitral regurgitation causing severe dysphagia resolved with isolated aortic valve replacement: an unusual case with few learning points. Indian J Clin Anaesth 2019;6(2):298-301. 